\title{
Weakly Semiprime Segments in Ordered Semigroups
}

\author{
Panuwat Luangchaisri and Thawhat Changphas * \\ Department of Mathematics, Faculty of Science, Khon Kaen University, Khon Kaen 40002, Thailand \\ * Correspondence: thacha@kku.ac.th
}

Received: 11 July 2019; Accepted: 28 August 2019; Published: 1 September 2019

\begin{abstract}
Let $P_{2} \subset P_{1}$ be a pair of weakly semiprime ideals of an ordered semigroup $(S, \cdot, \leq)$. Then, the pair $P_{2} \subset P_{1}$ is called a weakly semiprime segment of $S$ if $\bigcap_{n \in \mathbb{N}} I^{n} \subseteq P_{2}$ for all ideals $I$ of $S$ such that $P_{2} \subset I \subset P_{1}$. In this paper, we classify weakly semiprime segments of an ordered semigroup into four types; those that are simple, exceptional, Archimedean, and decomposable.
\end{abstract}

Keywords: weakly semiprime; prime; segment; ordered semigroup

\section{Introduction}

The theory of ideals in rings has been widely studied. Although the ideal theories of semigroups and of rings are similar, the axioms for semigroups are simpler than rings, whence there it is possible to obtain generally only weaker structural results.

The above paragraph clarifies the relationship between the ideal theory of semigroups and that of rings. In fact, a semigroup is a specific case of ordered semigroups, then the ideal theory of rings is always investigated on semigroups, and this is investigated in ordered semigroups later. For instance, Brungs and Törner [1] studied prime segments on rings. Then, this concept was moved to semigroups by Ferrero, Mazurek, and Sant'Ana [2] . Finally, it was extended to ordered semigroups by Luangchaisri, Changphas, and Mazurek in [3].

On a ring $(R,+, \cdot)$, a semiprime segment is a pair $P_{2} \subset P_{1}$ of semiprime ideals of $R$ such that $\bigcap_{n \in \mathbb{N}} I^{n} \subseteq P_{2}$ for all ideals $I$ of $R$ with $P_{2} \subset I \subset P_{1}$. This concept was introduced by R.Mazurek and G. Törner in [4]. They showed that semiprime segments on a ring can be classified into four types consisting of simple, exceptional, Archimedean, or decomposable. Moreover, they classified a semiprime segment $P_{2} \subset P_{1}$ when $P_{1}$ is a comparizer right ideal of $R$, and they obtained that this semiprime segment occurred only in three types consisting of simple, exceptional, or Archimedean. Hence, the purpose of this paper is to describe and classify weakly semiprime segments on an ordered semigroup as a result in [4].

An ordered semigroup $(S, \cdot, \leq)$ consists of a semigroup $(S, \cdot)$ together with a partial order $\leq$ that is compatible with the semigroup operation, i.e., for any $x, y, z \in S$,

$$
x \leq y \Rightarrow z x \leq z y, x z \leq y z .
$$

For $\varnothing \neq A, B \subseteq S$, the set product $A B$ is defined as follows:

$$
A B=\{a b \mid a \in A, b \in B\}
$$

and we define $(A]$ by:

$$
(A]=\{s \in S \mid s \leq a \text { for some } a \in A\} .
$$

For an ordered semigroup $(S, \cdot, \leq)$, it is observed (see [5]) that the following assertions hold:

1. $A \subseteq(A] ;$ 
2. $A \subseteq B \Rightarrow(A] \subseteq(B]$;

3. $(A](B] \subseteq(A B]$;

4. $\quad((A]]=(A]$.

Let $(S, \cdot, \leq)$ be an ordered semigroup, and let $A$ be a nonempty subset of $S$. Then, $A$ is called a right ideal of $S$ if $A S \subseteq A$; and $(A]=A$. We say that $A$ is a left ideal of $S$ if $S A \subseteq A$; and $(A]=A$. Finally, $A$ is an ideal of $S$ if it is both a left and a right ideal of $S$. It is easy to observe that:

1. If $A$ and $B$ are ideals of $S$, so are $(A B]$ and $A \cap B$;

2. $\quad(S a S]$ is an ideal of $S$ for all $a \in S$.

Next, we recall the concepts of prime ideals, weakly prime ideals, semiprime ideals, and weakly semiprime ideals of an ordered semigroup $(S, \cdot, \leq)$.

Definition 1. [5] Let $P$ be an ideal of an ordered semigroup $(S, \cdot, \leq)$. Then:

1. $P$ is prime if for all $A, B \subseteq S, A B \subseteq P$ implies $A \subseteq P$ or $B \subseteq P$;

2. $P$ is weakly prime if for all ideals $A, B$ of $S, A B \subseteq P$ implies $A \subseteq P$ or $B \subseteq P$;

3. $P$ is semiprime if for each $A \subseteq S, A^{2} \subseteq P$ implies $A \subseteq P$;

4. $P$ is weakly semiprime if for each ideal $A$ of $S, A^{2} \subseteq P$ implies $A \subseteq P$.

Remark 1. Let $P$ be an ideal of an ordered semigroup $(S, \cdot, \leq)$. Then:

1. $P$ is prime if for all $a, b \in S, a b \in P$ implies $a \in P$ or $b \in P$;

2. $P$ is weakly prime if for all $a, b \in S, a S b \subseteq P$ implies $a \in P$ or $b \in P$;

3. $P$ is semiprime if for each $a \in S, a^{2} \in P$ implies $a \in P$;

4. $P$ is weakly semiprime if for each $a \in S, a S a \subseteq P$ implies $a \in P$.

Lemma 1. [3] An ideal $P$ of an ordered semigroup $(S, \cdot, \leq)$ is prime if and only if $P$ is both semiprime and weakly prime.

Lemma 2. An ideal I of an ordered semigroup $(S, \cdot, \leq)$ is weakly semiprime if and only if it is the intersection of all weakly prime ideals of $S$ containing $I$.

Proof. Assume that $I$ is weakly semiprime. Let $F$ be the intersection of all weakly prime ideals of $S$ containing I. Suppose that there exists $x_{0} \in F \backslash I$. Then:

$$
x_{0} s_{0} x_{0} \notin I
$$

for some $s_{0} \in S$. Let:

$$
x_{1}=x_{0} s_{0} x_{0} .
$$

Then, there exists $s_{1} \in S$ such that:

$$
x_{1} s_{1} x_{1} \notin I .
$$

Continuing in a similar manner for $n \geq 2$, then we obtain the set:

$$
A=\left\{x_{0}, x_{1}, x_{2}, \ldots\right\}
$$

Let $Q$ be the union of all ideals of $S$ not containing any element of $A$. Then, $Q \neq \varnothing$ since $I \subseteq Q$. Let $J, K$ be ideals of $S$ such that $J K \subseteq Q$. If neither $J \subseteq Q$ nor $K \subseteq Q$, then:

$$
x_{n} \in J \text { and } x_{m} \in K
$$


for some $n, m \in \mathbb{N}$. In the case $n \geq m$, we obtain that:

$$
x_{n+1} \in x_{n} S x_{n} \subseteq x_{n} S x_{m} \subseteq J K \subseteq Q .
$$

This is a contradiction. The case $m \geq n$ can be proven similarly. Thus, $Q$ is a weakly prime ideal of $S$ containing $I$ and $x_{0} \notin Q$. This contradicts $x_{0} \in F$. Hence, $F=I$. The opposite direction is clear.

\section{Results}

Let $(S, \cdot, \leq)$ be an ordered semigroup. An element $e$ of $S$ is called an identity if $e a=a=a e$ for every $a \in S$. This section introduces a weakly semiprime segment on $S$, which is defined as a semiprime segment on rings. Then, we classify weakly semiprime segments on an ordered semigroup $S$ according to a classification of semiprime segments on rings. Moreover, we give an example of each type of weakly semiprime segments on $S$ to show that every type of this can occur. From now on, let $(S, \cdot, \leq)$ be an ordered semigroup with identity.

Definition 2. A pair $P_{2} \subset P_{1}$ of weakly semiprime ideals is called a weakly semiprime segment if for every ideal I of $S$ such that $P_{2} \subset I \subset P_{1}$,

$$
\bigcap_{n \in \mathbb{N}}\left(I^{n}\right] \subseteq P_{2}
$$

Example 1. Consider a semigroup $\mathbb{Z}_{30}$ under the usual multiplication. It is clear that $\overline{0}$ and $\overline{6} \mathbb{Z}_{30}$ are semiprime ideals of $\mathbb{Z}_{30}$. Since there are no ideals properly contained in the interval $\overline{0} \subset \overline{6} \mathbb{Z}_{30}$, we have that $\overline{0} \subset \overline{6} \mathbb{Z}_{30}$ is a semiprime segment of a semigroup $\mathbb{Z}_{30}$.

Definition 3. Let $P_{2} \subset P_{1}$ be a weakly semiprime segment of an ordered semigroup $(S, \cdot, \leq)$. Then, $P_{2} \subset P_{1}$ is simple if there is no further ideals of $S$ between $P_{2}$ and $P_{1}$, and $P_{2}$ is comparable with each ideal of $S$ contained in $P_{1}$.

Definition 4. Let $P_{2} \subset P_{1}$ be a weakly semiprime segment of an ordered semigroup $(S, \cdot, \leq)$. Then. $P_{2} \subset P_{1}$ is exceptional if there exists a weakly semiprime ideal $Q$ of $S$ such that $P_{2} \subset Q \subset P_{1}$ and $Q$ is comparable with each ideal of $S$ contained in $P_{1}$.

Definition 5. Let $P_{2} \subset P_{1}$ be a weakly semiprime segment of an ordered semigroup $(S, \cdot, \leq)$. Then, $P_{2} \subset P_{1}$ is Archimedean if for each $a \in P_{1} \backslash P_{2}$, there exists an ideal I of $S$ with $a \in I \subseteq P_{1}$ and $\bigcap_{n \in \mathbb{N}}\left(I^{n}\right] \subseteq P_{2}$.

Definition 6. Let $P_{2} \subset P_{1}$ be a weakly semiprime segment of an ordered semigroup $(S, \cdot, \leq)$. Then, $P_{2} \subset P_{1}$ is decomposable if $P_{2} \subset P_{1}$ is not Archimedean and $P_{1}=A \cup B$ where $A, B$ are ideals of $S$ properly contained in $P_{1}$.

The following examples show various types of weakly semiprime segments on ordered semigroups.

Example 2. Let $S=\left\{\frac{1}{n} \mid n \in \mathbb{N}, n \geq 2\right\} \cup\{0\}$ be a semigroup under the usual multiplication. Let $P_{1}=S$ and $P_{2}=\{0\}$. We can observe that $P_{2} \subset P_{1}$ is a semiprime segment of $S$. For each $a \in P_{1} \backslash P_{2}$, we have that $P_{1}$ is an ideal of $S$ containing $a$, which satisfies the condition:

$$
\bigcap_{n=1}^{\infty} P_{1}^{n} \subseteq P_{2}
$$

Thus, a semiprime segment $P_{2} \subset P_{1}$ is Archimedean. 
Example 3. Let us consider the semigroup with identity $S=\left\{\frac{1}{n} \mid n \in \mathbb{N}\right\} \cup\{0\}$ under the usual multiplication. If we define $P_{1}=S$ and $P_{2}=\{0\}$, then a pair $P_{2} \subset P_{1}$ is a semiprime segment of $S$. Given $I=\left\{\frac{1}{n} \mid n \in \mathbb{N}, n \geq 2\right\} \cup\{0\}$, then $I$ is a semiprime ideal of $S$ and $P_{2} \subset I \subset P_{1}$. Thus, a semiprime segment $P_{2} \subset P_{1}$ is exceptional.

Example 4. Let $S=\left\{0, x, a, b, b^{2}, b^{3}, \ldots\right\}$, and define a binary operation on $S$ as the following table:

\begin{tabular}{cccccccc}
\hline$\cdot$ & 0 & $x$ & $a$ & $b$ & $b^{2}$ & $b^{3}$ & $\ldots$ \\
0 & 0 & 0 & 0 & 0 & 0 & 0 & $\ldots$ \\
$x$ & 0 & $x$ & 0 & 0 & 0 & 0 & $\ldots$ \\
$a$ & 0 & 0 & $a$ & $b$ & $b^{2}$ & $b^{3}$ & $\ldots$ \\
$b$ & 0 & 0 & $b$ & $b^{2}$ & $b^{3}$ & $b^{4}$ & $\ldots$ \\
$b^{2}$ & 0 & 0 & $b^{2}$ & $b^{3}$ & $b^{4}$ & $b^{5}$ & $\ldots$ \\
$b^{3}$ & 0 & 0 & $b^{3}$ & $b^{4}$ & $b^{5}$ & $b^{6}$ & $\ldots$ \\
$\vdots$ & $\vdots$ & $\vdots$ & $\vdots$ & $\vdots$ & $\vdots$ & $\vdots$ & $\ddots$ \\
\hline
\end{tabular}

Then, $S$ is a semigroup. Let $P_{1}=S$ and $P_{2}=\{0, x\}$. It is clear that a pair $P_{2} \subset P_{1}$ is a semiprime segment of $S$. We can observe that $I=\left\{0, a, b, b^{2}, \ldots\right\}$ and $S$ are only ideals of $S$ containing $b$. Since:

$$
\bigcap_{n=1}^{\infty} I^{n} \nsubseteq P_{2} \text { and } \bigcap_{n=1}^{\infty} S^{n} \nsubseteq P_{2}
$$

it follows that $P_{2} \subset P_{1}$ is not Archimedean. Moreover,

$$
P_{1}=\{0, x\} \cup\left\{0, a, b, b^{2}, b^{3}, \ldots\right\}
$$

implies that $P_{2} \subseteq P_{1}$ is decomposable.

Theorem 1. Let $P_{2} \subset P_{1}$ be a weakly semiprime segment of an ordered semigroup $(S, \cdot, \leq)$. Then, exactly one of the following possibilities occurs:

1. There are no further ideals of $S$ between $P_{2}$ and $P_{1}$, and $P_{2}$ is comparable with each ideal of $S$ contained in $P_{1}$;

2. There exists a weakly semiprime ideal $Q$ of $S$ such that $P_{2} \subset Q \subset P_{1}$, and $Q$ is comparable with each ideal of $S$ contained in $P_{1}$;

3. $\quad\left(P_{1} a S \cup S a P_{1}\right] \subset(S a S]$ for all $a \in P_{1} \backslash P_{2}$;

4. $P_{1}=A \cup B$ for some ideals $A, B$ properly contained in $P_{1}$.

Proof. Let $a \in P_{1} \backslash P_{2}$. First of all, assume that $\left(P_{1}^{2} \cup P_{2}\right] \subset P_{1}$. If $a \in\left(P_{1} a S \cup S a P_{1}\right]$, then:

$$
a \in\left(P_{1}^{n} a S^{n} \cup S^{n} a P_{1}^{n}\right] \subseteq\left(P_{1}^{n}\right]
$$

for all positive integers $n$. This implies that:

$$
a \in \bigcap_{n=1}^{\infty}\left(P_{1}^{n}\right]=\bigcap_{n=1}^{\infty}\left(P_{1}^{2 n}\right] \subseteq \bigcap_{n=1}^{\infty}\left(\left(P_{1}^{2} \cup P_{2}\right)^{n}\right] \subseteq P_{2} .
$$

This contradicts $a \notin P_{2}$. Thus, $a \notin\left(P_{1} a S \cup S a P_{1}\right]$, and so, $\left(P_{1} a S \cup S a P_{1}\right] \subset(S a S]$. Next, assume that $\left(P_{1}^{2} \cup P_{2}\right]=P_{1}$. Let $M$ be the union of all ideals of $S$ properly contained in $P_{1}$. Then:

$$
P_{2} \subseteq M \subseteq P_{1}
$$

If $P_{2}=M$, then (1) occurs. Otherwise, we have only two cases to consider: 
Case 1. $P_{2} \subset M \subset P_{1}$. Then, $M$ is a weakly semiprime ideal of $S$, and (2) occurs. Indeed: Let $A$ be an ideal of $S$ such that $A^{2} \subseteq M$. Then, $A^{2} \subseteq P_{1}$, which implies $A \subseteq P_{1}$ since $P_{1}$ is weakly semiprime. If $A \subset P_{1}$, we have that $A \subseteq M$. Otherwise,

$$
P_{1}=\left(P_{1}^{2} \cup P_{2}\right]=\left(A^{2} \cup P_{2}\right] \subseteq M .
$$

This implies that $A \subseteq M$.

Case 2. $P_{2} \subset M=P_{1}$. For each $b \in P_{1}$, we have that $(S b S] \subset P_{1}$ since $M=P_{1}$. Suppose that (3) does not occur. Then, there exists $c \in P_{1} \backslash P_{2}$ such that $\left(P_{1} c S \cup S c P_{1}\right]=(S c S]$. That is:

$$
c \in(p c S \cup S c q]
$$

for some $p, q \in P_{1}$. Set $I=(S p S \cup S q S]$. Then, $I$ is an ideal of $S$ and:

$$
c \in(p c S \cup S c q] \subseteq(S p S c S \cup S c S q S] \subseteq(I c S \cup S c I] .
$$

If $\left(I \cup P_{2}\right] \subset P_{1}$, then:

$$
c \in \bigcap_{n=1}^{\infty}\left(I^{n}\right] \subseteq \bigcap_{n=1}^{\infty}\left(\left(I \cup P_{2}\right)^{n}\right] \subseteq P_{2},
$$

a contradiction. Thus, $P_{1}=I \cup P_{2}$.

By using Theorem 1 , a weakly semiprime segment $P_{2} \subset P_{1}$ of an ordered $\operatorname{semigroup}(S, \cdot, \leq)$ can be classified into four types.

Theorem 2. If $P_{2} \subset P_{1}$ is a weakly semiprime segment of an ordered semigroup $(S, \cdot, \leq)$, then exactly one of the following possibilities occurs:

1. $\quad P_{2} \subset P_{1}$ is simple;

2. $\quad P_{2} \subset P_{1}$ is exceptional;

3. $P_{2} \subset P_{1}$ is Archimedean;

4. $P_{2} \subset P_{1}$ is decomposable.

Proof. Assume that a pair $P_{2} \subset P_{1}$ of semiprime ideals of $S$ is a semiprime segment of $S$. By Theorem 1 , there are four cases to consider. It is clear that if (1), (2), or (4) occurs, then a semiprime segment $P_{2} \subset P_{1}$ is simple, exceptional, or decomposable, respectively. Assume that:

$$
\left(P_{1} a S \cup S a P_{1}\right] \subset(S a S]
$$

for all $a \in P_{1} \backslash P_{2}$. If $P_{2} \subset P_{1}$ is not Archimedean, then there is $b \in P_{1} \backslash P_{2}$ such that for all ideals $I$ of $S$ with $b \in I \subseteq P_{1}$,

$$
\bigcap_{n=1}^{\infty}\left(I^{n}\right] \nsubseteq P_{2} .
$$

Suppose that $\left((S b S)^{2}\right] \cup P_{2} \subset P_{1}$. Then, we have that:

$$
\bigcap_{n=1}^{\infty}\left((S b S)^{n}\right]=\bigcap_{n=1}^{\infty}\left((S b S)^{2 n}\right] \subseteq \bigcap_{n=1}^{\infty}\left(\left((S b S)^{2} \cup P_{2}\right)^{n}\right] \subseteq P_{2} .
$$

This contradicts $P_{2} \subset P_{1}$ being not Archimedean. Thus, $\left((S b S)^{2}\right] \cup P_{2}=P_{1}$. By assumption, we obtain that:

$$
\left((S b S)^{2}\right] \subseteq\left(P_{1} b S\right] \subset(S b S] \subseteq P_{1} .
$$

Hence, a semiprime segment $P_{2} \subset P_{1}$ is decomposable. It is obvious that every type of a semiprime segment is mutually exclusive. 
Definition 7. Let $R$ be a right ideal of an ordered semigroup $(S, \cdot, \leq)$. Then, $R$ is said to be a waist of $S$ if for all right ideal $A$ of $S$, either $R \subseteq A$ or $A \subset R$.

Definition 8. Let $R$ be a proper right ideal of an ordered semigroup $(S, \cdot, \leq)$. Then, $R$ is said to be a comparizer right ideal if for any $a, b \in S$, either $(a S] \subseteq(b S]$ or $(b R] \subseteq(a S]$.

Lemma 3. Let $R$ be a right ideal of an ordered semigroup $(S, \cdot, \leq)$. Then, $R$ is a comparizer of $S$ if and only if for each right ideal $A, B$ of $S$,

$$
A \nsubseteq B \text { implies }(B R] \subset A \text {. }
$$

Proof. Assume that $R$ is a comparizer right ideal of $S$. Let $A, B$ be right ideals of $S$ such that $A \nsubseteq B$. Then, there exists $a \in A$ such that:

$$
(a S] \nsubseteq(b S]
$$

for all $b \in B$. By assumption, we obtain that:

$$
(b R] \subseteq(a S] \subseteq A
$$

Thus,

$$
(B R] \subseteq A .
$$

Conversely, let $a, b \in S$ be such that $(a S] \nsubseteq(b S]$. Then:

$$
(b R] \subseteq(b S R]=((b S] R] \subseteq(a S] .
$$

Thus, $R$ is a comparizer right ideal of $S$.

Theorem 3. For any ordered semigroup $(S, \cdot, \leq)$, the following right ideals are comparizer right ideals.

1. The union of a family of comparizer right ideals of $S$;

2. $\quad$ The ideal $I(R)$ of $S$ generated by a comparizer right ideal $R$ of $S$;

3. A right ideal of $S$ contained in a comparizer right ideal of $S$.

Proof. (1) Let $C=\cup\left\{A_{\alpha} \mid \alpha \in \Lambda\right\}$, where $A_{\alpha}$ is a comparizer right ideal of $S$. Let $a, b \in S$ such that $(a S] \nsubseteq(b S]$. Then:

$$
\left(b A_{\alpha}\right] \subseteq(a S]
$$

for all $\alpha \in \Lambda$. That is:

$$
(b C]=\left(b\left(\bigcup_{\alpha \in \Lambda} A_{\alpha}\right)\right]=\left(\bigcup_{\alpha \in \Lambda} b A_{\alpha}\right] \subseteq(a S] .
$$

Thus, $C$ is a comparizer right ideal of $S$.

(2) Let $R$ be a comparizer right ideal of $S$, and let $A, B$ be right ideals of $S$ such that $A \nsubseteq B$. Then:

$$
\begin{aligned}
(B I(R)] & =(B(S R S]] \\
& =(B S R S] \\
& =(B R] \\
& \subseteq(A] \\
& =A .
\end{aligned}
$$

Therefore, $I(R)$ is a comparizer right ideal of $S$.

(3) Let $R^{\prime}$ be a right ideal of $S$ contained in a comparizer right ideal $R$ of $S$. Let $a, b \in S$ be such that:

$$
(a S] \not \subset(b S] \text {. }
$$


Then:

$$
\left(b R^{\prime}\right] \subseteq(b R] \subseteq(a S] .
$$

Thus, $R^{\prime}$ is a comparizer right ideal of $S$.

Theorem 4. Let $R$ be a comparizer right ideal of an ordered semigroup $(S, \cdot, \leq)$. Then, the following statements hold:

1. If $R=\left(R^{2}\right]$, then $R$ is a waist of $S$;

2. If $P$ is a weakly prime ideal of $S$ such that $R \nsubseteq P$, then $P$ is a waist of $S$ and $P \subset R$.

Proof. (1) Assume that $R=\left(R^{2}\right]$. Let $A$ be a right ideal of $S$ such that $A \nsubseteq R$. Then:

$$
R=\left(R^{2}\right] \subseteq A
$$

implies that $R$ is a waist of $S$.

(2) Assume that $P$ is a weakly prime ideal of $S$ such that $R \nsubseteq P$. Let $A$ be an ideal of $S$ such that $A \nsubseteq P$. Then, $(A R] \nsubseteq \subset P$ since $P$ is weakly prime. It follows that $P \subset A$ since $R$ is a comparizer right ideal of $S$. Thus, $P$ is a waist of $S$.

Note that the set of weakly prime ideals of $S$ contained in a comparizer ideal is linearly ordered by inclusion.

Theorem 5. Let $R$ be a comparizer right ideal of an ordered semigroup $(S, \cdot, \leq)$. If $R$ is an ideal of $S$, then for each ideal $Q$ of $S$ such that $Q \subset R, Q$ is weakly semiprime if and only if $Q$ is weakly prime.

Proof. Assume that $Q$ is weakly semiprime. Then, $Q=\bigcap_{\alpha \in \Lambda} P_{\alpha}$, where $P_{\alpha}$ is a weakly prime ideal of $S$ containing $Q$. By Theorem 4, there exists $\alpha \in \Lambda$ such that $P_{\alpha} \subset R$. Let $A, B$ be ideals of $S$ such that $A B \subseteq Q$. If neither $A \subseteq Q$ nor $B \subseteq Q$, then $A \nsubseteq P_{\beta}$ and $B \nsubseteq P_{\gamma}$ for some $\beta, \gamma \in \Lambda$. Now, we have four cases to consider:

Case 1: If $R \subseteq P_{\beta}$ and $R \subseteq P_{\gamma}$, then:

$$
A \not \subset P_{\beta} \supseteq R \supset P_{\alpha}
$$

and:

$$
B \nsubseteq P_{\gamma} \supseteq R \supset P_{\alpha} .
$$

These contradict $P_{\alpha}$ being weakly prime.

Case 2: If $P_{\beta} \subset R \subseteq P_{\gamma}$, then:

$$
A \nsubseteq P_{\beta}
$$

and:

$$
B \nsubseteq P_{\gamma} \supset P_{\beta}
$$

These contradict $P_{\beta}$ being weakly prime.

Case 3: If $P_{\gamma} \subset R \subseteq P_{\beta}$, we can prove this similarly to Case 2 .

Case 4: If $P_{\gamma} \subset R$ and $P_{\beta} \subset R$, then we have:

$$
P_{\gamma} \subseteq P_{\beta} \text { or } P_{\beta} \subseteq P_{\gamma} .
$$

This case does not occur as Cases 2 and 3 .

Thus, $A \subseteq Q$ or $B \subseteq Q$. Therefore, $Q$ is weakly prime. The opposite direction is clear.

Theorem 6. Let $P_{2} \subset P_{1}$ be a weakly semiprime segment of an ordered semigroup $(S, \cdot, \leq)$. If $P_{1}$ is a comparizer right ideal of $S$, then one of the following possibilities occurs:

1. The weakly semiprime segment $P_{2} \subset P_{1}$ is simple. In this case, $P_{2}$ is a weakly prime waist ideal of $S$; 
2. The weakly semiprime segment $P_{2} \subset P_{1}$ is exceptional. In this case, there exists a weakly prime waist ideal $Q$ of $S$ with $P_{2} \subset Q \subset P_{1}$ such that there are no further ideals between $Q$ and $P_{1}$. Moreover, $P_{2}$ is a weakly prime waist ideal of $S$;

3. The weakly semiprime segment $P_{2} \subset P_{1}$ is Archimedean. In this case, $P_{2}$ is a weakly prime waist ideal of $S$.

Proof. Assume that a weakly semiprime segment $P_{1}$ is a comparizer right ideal of $S$. By Theorem 5, we have that $P_{2}$ is a weakly prime ideal of $S$. Moreover, $P_{2}$ is also a waist of $S$ by Theorem 4 . By using Theorem 1, there are four cases that have to be proven. It is clear that Theorem 1(1) implies (1). Assume that Theorem 1(2) holds. Then there is a weakly semiprime ideal $Q$ with $P_{2} \subset Q \subset P_{1}$. According to the proof of Theorem 2, we obtain immediately that there are no further ideals between $Q$ and $P_{1}$. Thus, this case implies that (2) holds.

Next, assume that Theorem 1(3) or Theorem 1(4) occurs. If a weakly semiprime segment $P_{2} \subset P_{1}$ is not Archimedean, by the proof of Theorem 2, then $P_{2} \subset P_{1}$ is decomposable. That is $P_{1}=A \cup B$ for some ideals $A, B$ of $S$ properly contained in $P_{1}$. Since $P_{2}$ is a waist of $S$, then there are four cases to consider:

( $\alpha) \quad P_{2} \subset A$ and $P_{2} \subset B$. Then, we obtain immediately that $P_{2} \subset P_{1}$ is Archimedean. This contradicts the hypothesis. Therefore, this case does not occur.

( $\beta) \quad P_{2} \subset A$ and $B \subseteq P_{2}$. For each $a \in P_{1} \backslash P_{2}$, we have that $a \in A \subseteq P_{1}$ and $\bigcap_{n=1}^{\infty} A^{n} \subseteq P_{2}$. This implies that a weakly semiprime segment $P_{2} \subset P_{1}$ is Archimedean. This is a contradiction.

$(\gamma) A \subset P_{2}$ and $P_{2} \subseteq B$. It can be proven similarly to the case $(\beta)$ that this case does not occur.

( $\delta) \quad A \subseteq P_{2}$ and $B \subseteq P_{2}$. Then, $P_{1}=A \cup B=P_{2}$. This case does not occur.

Hence, if Theorem 1(3) or Theorem 1(4) occurs, we can infer that a weakly semiprime segment $P_{2} \subseteq P_{1}$ is Archimedean.

\section{Conclusions}

Weakly semiprime segments defined on an ordered semigroup $(S, \cdot, \leq)$ can be classified into four types according to a classification of semiprime segments on rings. In particular, if a weakly semiprime segment $P_{2} \subset P_{1}$ has the property that $P_{1}$ is a comparizer right ideal of $S$, then a weakly semiprime segment $P_{2} \subset P_{1}$ is not decomposable. This implies that a weakly prime segment $P_{2} \subset P_{1}$ can be only simple, exceptional, or Archimedean.

Author Contributions: All authors have cooperated in the preparation of this work. Conceptualization, P.L. and T.C.; methodology, P.L. and T.C.; validation, T.C.; formal analysis, P.L. and T.C.; writing, original draft preparation, P.L.; writing, review and editing, P.L. and T.C.; project administration, P.L. and T.C.

Funding: This research received no external funding.

Acknowledgments: P. Luangchaisri is supported by the Research Fund for Supporting Lecturer to Admit High Potential Student to Study and Researchfor His Expert Program Year 2016. T. Changphas is supported by Grant Number 6200056 of the National Research Council of Thailand (NRCT).

Conflicts of Interest: The authors declare no conflict of interest.

\section{References}

1. Brungs, H.H.; Törner, G. Ideal theory of right cones and associated rings. J. Algebra 1998, 210, 145-164. [CrossRef]

2. Ferrero, M.; Mazurek, R.; Sant'Ana, A. On right chain semigroups. J. Algebra 2005, 292, 574-584. [CrossRef]

3. Changphas, T.; Luangchaisri, P.; Mazzurek, R. On right chain ordered semigroups. Semigroup Forum 2018, 96, 523-535. [CrossRef] 
4. Mazzurek, R.; Torner, G. On semiprime segments of rings. J. Aust. Math. Soc. 2006, 80, 263-272. [CrossRef]

5. Kehayopulu, N. On prime, weakly prime ideals in ordered semigroups. Semigroup Forum 1992, 44, 341-346. [CrossRef]

(c)

(C) 2019 by the authors. Licensee MDPI, Basel, Switzerland. This article is an open access article distributed under the terms and conditions of the Creative Commons Attribution (CC BY) license (http:// creativecommons.org/licenses/by/4.0/). 